\title{
An optimization algorithm for hybrid control of robot force/position
}

\author{
Sun Haibo ${ }^{1, a, *}$, Zhang Qiuhao ${ }^{1, b}$ \\ ${ }^{1}$ School of information science and Engineering, Shenyang University of Technology, Shen West \\ Road Shenyang economic and Technological Development Zone No. 111, Shen Yang, China \\ ${ }^{2}$ Control theory and control engineering, Shenyang University of Technology, Shen West Road \\ Shenyang economic and Technological Development Zone No. 111, Shen Yang, China \\ a1064446256@qq.com, 56752376@qq.com \\ *Corresponding author
}

Keywords: Hybrid force / position control, Redundant robot, Zero space vector.

\begin{abstract}
Through the research of robot system of hybrid force/position control for the robot, In view of the robot in contact with the environment, Force control in a certain direction, the position deviation in the direction is ignored. Therefore, when the robot position control, the equivalent of redundant robots. In this paper, a method is proposed to optimize the force of the zero space vector of redundant robot in Cartesian space. Finally, the experimental verification.
\end{abstract}

\section{Introduction}

Robot hybrid force/position control is the simultaneous tracking control of the contact force and position at the end of the robot.By using the orthogonal relation between the contact force and the position, the end motion of the robot is decomposed in Cartesian coordinates. In literature [1], the position control is used in the direction of non restraint, and the force is controlled in the constrained direction. This control method is based on force deviation control and is feedback control, The end effector of the robot can change with the expected value. However, the robot is a nonlinear time-varying system. The dynamic output of the joint is calculated by establishing a precise robot dynamics model. The joint driving force of the robot is affected by the inertia, Coriolis force and centripetal force. Another force/position hybrid control method is the robot impedance control. The design idea is to control the position of the robot to achieve the purpose of controlling the end force, to ensure that the robot in the constrained direction to maintain the desired contact force [2][3].This kind of control method is controlled according to the position deviation and is force feedforward control. Most industrial robots are based on joint space position control. Although this control method is affected by the dynamic model of the system, external interference and force measurement. However, the parameters of the ideal impedance model must be adjusted with the change of the environmental dynamics model parameters in the robot impedance control.

The hybrid force/position control method presented in this paper is based on the kinematic solution. The 6 degrees of freedom of the robot's end effector provide control for the 6 variables in 
the Cartesian space. When the degree of freedom of the end effector is constrained by the environment, in the direction of force control, then the direction of the position deviation should be ignored. At this point, the zero space position vector can be used to optimize the force control of the robot. Redundant degrees of freedom can improve the motion characteristics of the robot [4][5].Set the force difference performance index of the robot force/position hybrid control optimization. The advantage of this method is that it does not need to consider the dynamic model of the environment, the Coriolis force and the centripetal force. It is not necessary to calculate the joint output driving force so that the contact force can be a good tracking of the desired force.

\section{Redundancy based machine human optimal control based on zero space position vector}

When the robot is in contact with the environment, Force control in the direction perpendicular to the contact surface, At this point, the position deviation in the direction should be ignored.The position and orientation of the robot end effector can be represented by 5 ,vectors $n=6>5$ and $\operatorname{Rank}(J)=5$. At this point, the redundant robot can be used to solve the problem, and the redundant robot can optimize the force control.

The relationship between the operating speed and the joint velocity is:

$\dot{X}=J(q) \dot{q}$

In type $\dot{X} \in R^{m}, \dot{q} \in R^{n}$

The inverse kinematics solution is carried out to solve the joint velocity $\dot{q}$. For the redundant robot, the inverse solution of velocity can be given by $J^{+}(q)$ and $J(q)$ of zero space vectors:

$\dot{q}=J^{+}(q) \dot{X}+\left(I-J^{+} J\right) \dot{\phi}$

In the formula, $J^{+}$is the pseudo inverse of the Jacobi matrix, $J^{+} \in R^{n \times m} . I \in n \times n$ is order unit matrix, $\left(I-J^{+} J\right)$ is a mapping matrix of zero space $N(J)$ and $\dot{\phi} \in R^{n}$ is arbitrary vector.

This paper will be set a control index $\dot{\phi}, \dot{\phi}=\left[\begin{array}{c}F_{x} \\ F_{y} \\ F_{z} \\ m_{x} \\ m_{y} \\ m_{z}\end{array}\right]$. It contains three translation an three rotation vectors.

According to the contact characteristics between the robot and the environment, the difference of the force can be obtained:

$F_{c}=F-K_{e} X$

In the formula $F$ is a given force, $K_{e}$ is the elastic coefficient of representative system, it is constant. $X$ is represented the deviation of the robot motion.

$\dot{\phi}$ can complete the control in all directions. When the end effector is in contact with the environment, we can control the force in the constraint direction. The position deviation in the constraint direction should be ignored. So we can get expressions:

$J^{+}(q) \dot{X}=0$

Then we can know the joint angular velocity:

$\dot{q}=\left(I-J^{+} J\right) \dot{\phi}$ 


\section{Experimental verification}

As shown in Figure 1, the contact between the robot and the environment can be equivalent to a mass spring system, in which the stiffness coefficient of the spring is set to $\mathrm{Ke}=2$, the displacement of the motion is $\mathrm{x}$, and the desired force is set to $10 \mathrm{~N}$.

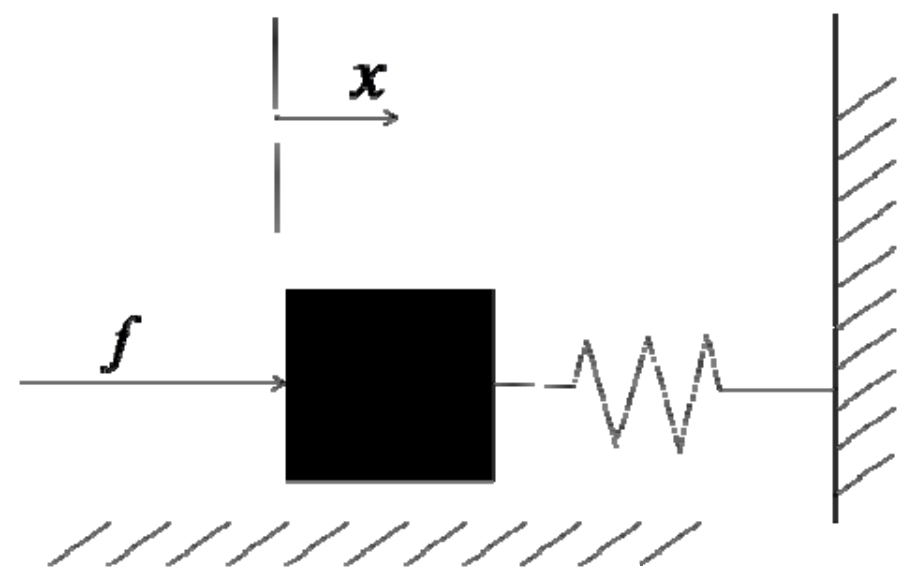

Figure 1Equivalent graph when robot and environment contact

The above algorithm theory can be used in MATLAB software. We step 0.01s,get 30 times to simulation. we can get the force difference curve in figure 2.It Described the corresponding relationship between $\mathrm{Fc}$ and $\mathrm{t}$.

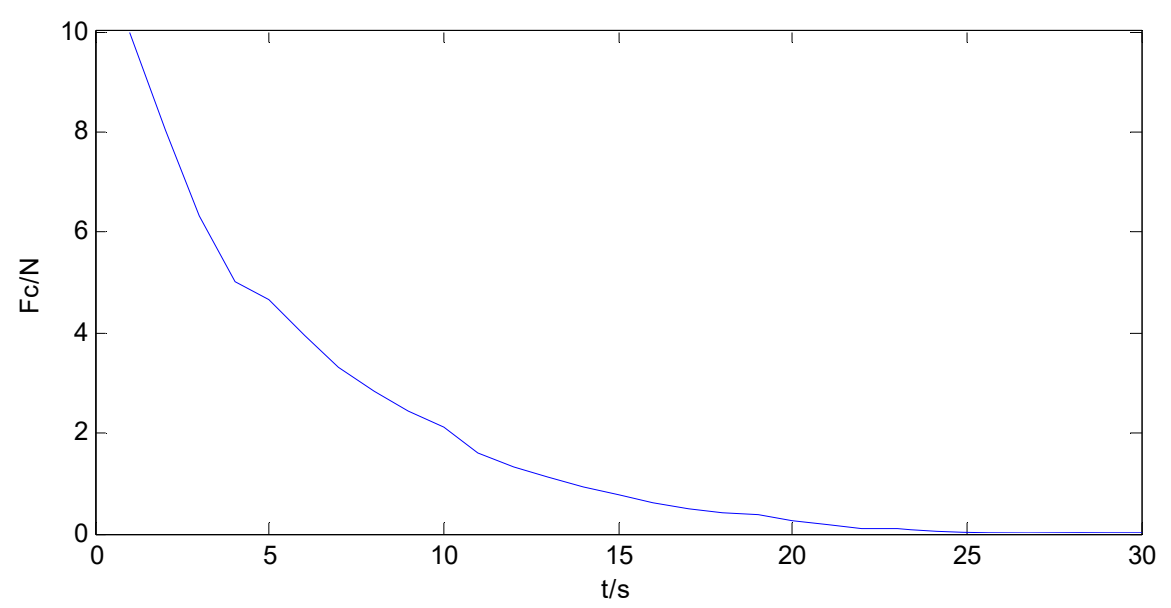

Figure 2 Image of the difference of force varies with time

We can know from the image: When the robot is initially, the force difference is the largest $10 \mathrm{~N}$, With the increase of time, the difference of force decreases gradually, The final force difference will tend to 0 N.Here,the end contact force of the robot will be the expected value. Experimental results show that in Cartesian space, It is feasible to use the zero space vector of redundant robot to optimize the force. 


\section{Conclusions}

In this paper, Aimed at the robot and the environment in contact with the situation. In Cartesian space, the feasibility of the optimization method is proved by using the zero space vector of redundant robot. The feasibility of the method is proved by experiments

\section{References}

[1] Bassi E,Benzi F,Capisani L M,et al.Hybrid position/force sliding mode control of a class of robotic manipulators[C]//48th IEEE Conference on Decision and Control held jointly with 2009 28th Chinese Control Conference.Shanghai,China:Institute of Electrical and Electrinics Engineers Inc.,2009:2966-2971.

[2] Seraji H,Colbaugh R.Force Tracking Impedance Control[J].International Journal of Robotics Research,1997,2(16):97-117.

[3] Cai Zixing, Xie Guanghan, Wu Zhaohui, et al. Direct realization of force / position adaptive fuzzy control in position control robot [J]. robot, (04): 23-30. (in Chinese)

[4] Marnni G , Kim J, Yuh J, et al. Algorithmic singularities avoidance in task-prio rity based controller for redundant manipulators[C].IEEE Int Conf on Intelligent Robots and Systems. Las Vegas, 2003:3570-3574.

[5] Zhang Y N, Wang J. Obstacle avoidance of redundant manipulators using a dual neural network [C].IEEE Int Conf on Robotics and Automation. Taipei, 2003: 2747-2752.

[6] Youlun Xiong. Robotics Machinery Industry Press.1992:255-259. 